\title{
Linking PCB Thermal Design Analysis Validation With Thermal NDT
}

\author{
V.N.Misale ${ }^{1}$ Samavi Vedullavalasa ${ }^{2}$ and S.Ganesan ${ }^{3}$
}

1. Scientist 'G', Dy. General Manager NDE, SRSPS, Liquid Propulsion systems

Centre, Sir C V Raman Hospital Road, HAL II Stage HPO, Bangalore 560008.

2. M.Tech Student, MVJ College of Engineering, Bangalore 560067.

3. Scientist 'SG', Product Development Manager Design, ISSTA Development

Lab,LPSC Campus Thermal Systems Group, ISAC, Bangalore 560017.

(Emails: vnmisale@gmail.com, samavipatnaik@gmail.com,

$$
\text { ganesan_cnj@techemail.com) }
$$

\begin{abstract}
Due to higher component packaging densities on Printed Circuit Boards (PCB), thermal design of PCB pose challenges to any heat transfer designer of PCB and electronic packages. PCB design proceeds on the strength of various assumptions in the predictions of temperatures. Some of the key contributing factors affecting temperature distribution in PCBs range from device power dissipations, their distribution in a board, layer-wise thermal conductivity and copper $(\mathrm{Cu})$ spread within a PCB, nature of drill-holes / thermal mass distribution and finally electrical heating effects affecting thermal performance of PCBs.
\end{abstract}

All the above factors bring uncertainty in prediction of temperatures in devices and inferior design generally results in de-lamination of PCB layers. Effects of DC drop due to passage of electric current inside $\mathrm{Cu}$-traces, impacts Joule heating effects inside traces of the board and lead to trace level fusing.

Even though several good analytical approaches exist today and good Mechanical computer Aided Engineering (MCAE) software tools for electronics packaging address many of the above design considerations for prediction of PCB temperatures using either finite elements / lumped techniques, a practicing thermal engineer would still find thermography as an effective technique to validate thermal results from any of these predictive methods.

In this paper we discuss the utilization of thermography as a NDT technique to consolidate and validate the findings for a PCB thermal Analysis and indicate how this this technique will enable a thermal engineer to identify the hotspots in the PCB's inner layers and improve thermal design.Experimental analysis support detection of hot spots and delamination, temperature measurements under transient and steady state conditions, isothemal maps, emissivity corrections and normalized thermal profiles generation, dual image techniques etc. Comparison is made between theoretical estimation of temperature during steady state operation and calibrated thermo graphic measurements for a typical PCB.

Key words: PCB Thermal design analysis, thermal performance design validation, PCB Evaluation, Thermography, Iso thermal maps, thermal line profile, dual image techniques 